\title{
APPLICATION OF DEEPLEARNING TECHNIQUES FOR COVID-19 DIAGNOSIS AND TREATMENT
}

\author{
Aruna Shankar \\ Assistant Professor, Department of Information Technology Management, \\ American University of Phnom Penh, Phnom Penh, Cambodia
}

\begin{abstract}
Covid-19 is an ongoing worldwide pandemic caused by severe acute respiratory syndrome coronavirus (Covid). The virus was first identified in late December 2019 in Wuhan China. As of (01/Jul/2021) 183 million people were infected with the Covid -19 and encountered moderate to severe respiratory sickness, 3.9 million died of this infection. Elderly people, adults, kids, and those with underlying medical conditions heart disease, diabetes, chronic respiratory disease, and cancer are at high risk of developing serious illnesses from covid-19. The current scenario documented the Multiple variants of the virus that causes Covid-19 which spreads faster than usual and remains a high threat to mankind. Despite the high-risk covid-19 variant, numerous ongoing clinical trials for diagnosis and treatment of coronavirus infection have been involved to treat the Patients. The current clinical trial tools are time-consuming, staggering a high cost, and requiring a well-equipped laboratory for analysis. Fast diagnostic methods can control, prevent the spread of covid-19 variants and reduce the workload of the physicians to better manage the patients. A computed tomography scan (CT) is the fastest method to diagnose patients with covid-19 variants. Nevertheless, the radiologist's performance is moderate in diagnosing the virus and time-consuming due to the overwhelmed patients. Discern the impact of covid-19 threats, computer science researchers have started using artificial intelligence techniques to detect the presence of covid-19 infection using CT scan. This study furnishes an elaborate response with various Deep Learning (DL) techniques of Artificial intelligence to combat the novel coronavirus. Furthermore, this study can improve the performance of introduced techniques towards the best responses in practical applications
\end{abstract}

Keywords Covid -19, Computed Tomography scan, Deep Learning, Artificial Intelligence.

\section{INTRODUCTION}

The coronavirus was first detected in late December 2019 and spread across the world briskly at high speed. Now it has affected almost all the country with cases around 185,455,633 people with infection and 4,010,632 were died, according to $07 / \mathrm{Jul} / 2021$ data recorded at coronavirus world meter since it was Confirmed a pandemic by the World health organization in March 2020. This covid-19 infection has created a tremendous impact mainly on health care and world economy. The covid-19 symptoms are fever, cough, vomiting, and diarrhea, as well as acute respiratory illness (Rodriguez-Palacios, 2020). The infection of coronavirus is transmitted predominantly via droplets of infected person. To prevent the spread of coronavirus, nations all over the world have declared lockdown, social distancing and isolation to limit the movement of the people.

The need of a protective vaccine is required to COVID-19 pandemic. The World Health Organization (WHO) has listed more than 200 COVID-19 vaccines were under development. The hope that preventive vaccines will control COVID19 is justified by the impact of vaccines on preventing disability and death from other infectious diseases. 15 vaccines out of 200 vaccines have been granted as emergency use by national regulatory authority. Five of those vaccines have been authorized for emergency or full use by WHO $(W H O, 2021$.). Usually all viruses, including the virus that causes COVID-19, change over time. Highest of the variant changes have little to no impact on the virus' properties. However, some differences may affect the virus's properties, such as how easily it spreads, the associated disease severity, or the performance of vaccines(Tracking SARS-CoV-2 Variants, n.d.).

Even Though the vaccine is in use, during late December 2020, there was an emergence of variants that posed an increased risk to global public health prompted the description of specific Variants. Viruses constantly change and become more diverse. Scientists monitor these changes, including changes to the spikes on the surface of the virus and alert the countries and public about any changes that may be needed to respond to the variant, and prevent its spread (About Variants of the Virus That Causes COVID-19|CDC, n.d.). global authorities are warned and also encouraged 
to strengthen surveillance and sequencing capacities and apply a systematic approach to provide a typical indication of the extent of transmission of covid variants based on the local context, and to detect unusual epidemiological events.

At the present time, real-time reverse transcription polymerase chain reaction (rRT-PCR) is used for diagnosing COVID-19. However, the limited sensitivity of rRT-PCR and the shortage of testing kits in an outbreak area increase the screening burden, and many infected people are thereby not isolated immediately. This accelerates the spread of COVID19 variants. On The Other Hand, due to the lack of medical resources, many affected patients cannot receive urgent treatment. In this situation, finding high-risk patients with a poorer prognosis for prior treatment and early prevention is important. Subsequently, fast diagnosis and finding high-risk patients with worse prognosis are very helpful for the control and management of COVID-19 variants.

In recent studies, radiological findings demonstrated that computed tomography (CT) has excellent indicative and predictive value for COVID-19. For example, CT showed much higher sensitivity than rRT-PCR in diagnosing COVID19 (Villalta et al., 2021).Even in asymptomatic patients, abnormalities and changes were observed in serial CT. As an universal diagnostic tool, CT is easy and fast to acquire without adding much cost. Building a sensitive diagnostic tool applying CT imaging can accelerate the diagnostic method and is complementary to RT-PCR. Still, predicting personalized prognosis using CT imaging can identify the prospective high-risk patients who are more likely to become severe and need urgent medical resources.

Deep learning in Artificial intelligence shows better performance to diagnose and treat the diseases like heart, diabetes, cancer and including covid-19. Combat against COVID-19, advanced technologies are playing an important role in the development of a smart healthcare system. COVID-19 Robots are employed to deliver food and medicine in hospital and drones are applied to disinfect streets from coronavirus (Kumar et al., 2020).Worldwide researchers are looking for fast diagnostic method for detecting and controlling coronavirus and to assist physicians to better manage patients in high workload conditions. Even If a laboratory test is the existing routine diagnostic tool, it is time-exhausting, levying a high cost and requiring a perfectly equipped research laboratory for analysis. Computed tomography (CT) has thus far become a rapid method to establish patients with COVID-19. Nevertheless, the performance of radiologists in finding of COVID-19 was modest. Suitably, supplementary inquiries are needed to improve the performance in spotting COVID19 (Ardakani et al., 2020). Deep learning is a technology of Artificial intelligence that can be able to diagnose the disease, predict the virus severity, track the infected person and also used to discover new drugs and medicine which helps the infected patient's recovery and utilize to reproduce the vaccine with better performance and reduce the human workload. This section concentrates on the remarkable contribution of Deep learning methods to combat against coronavirus. It also delivers a comprehensive review on application of deep learning techniques for covid-19 diagnosis and treatment. This study clearly describes with the challenges of existing system. The deep learning approaches have been categorized into two main categories namely, diagnosis and treatment. Furthermore, the diagnosis and treatment categories has been classified into sub fields like, medical imaging, virus severity, Tracking infected person and Drug discovery, Protein structure Vice versa. These applications are explained with the deep learning techniques throughout the study with possible future trends and with the conclusion. Deep learning focuses on its application with Covid-19 diagnosis and treatment to combat coronavirus. Figure 1 shows the various role of deep learning techniques to control COVID-19.

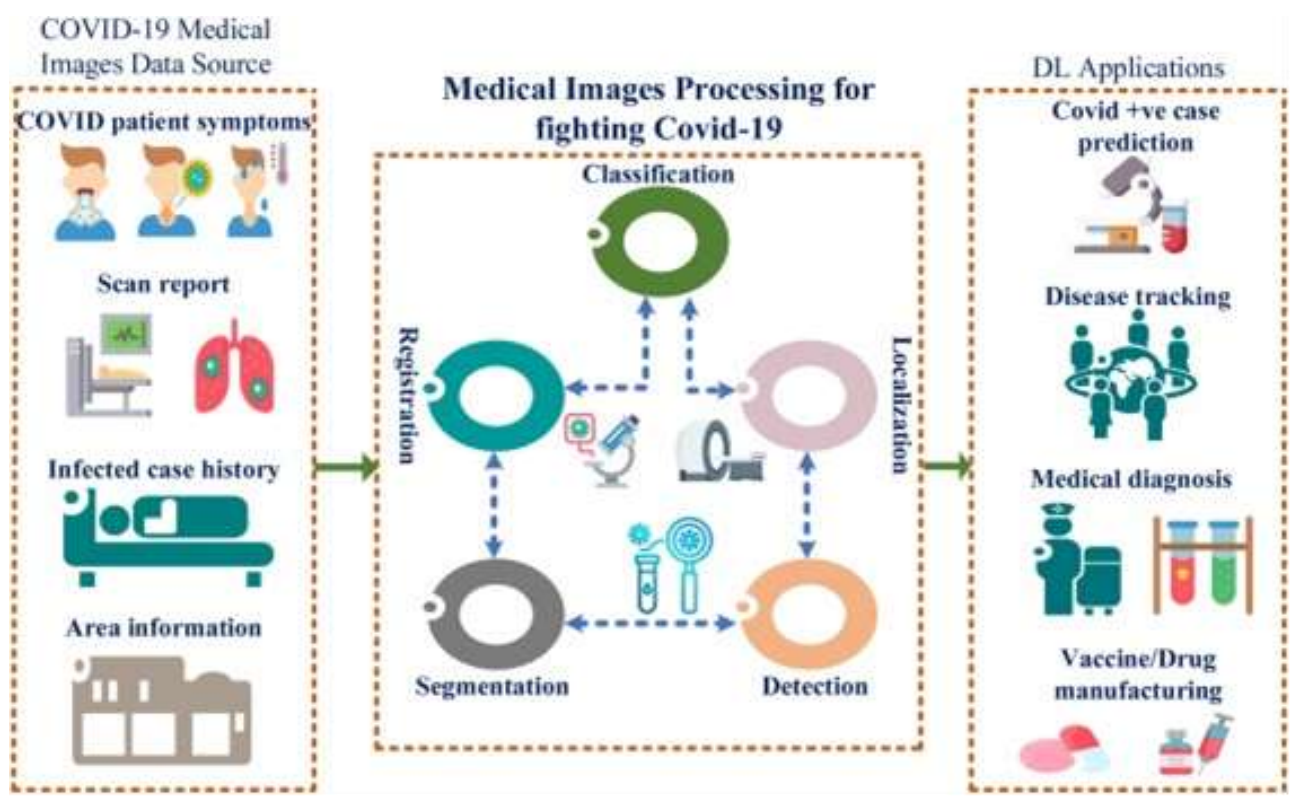

Figure 1. Application of Deep Learning in Medical imaging for combatting Covid-19 
Vol. 10, Issue 9, September 2021

DOI: $10.17148 /$ IJARCCE.2021.10904

\section{DEEP LEARNING IN MEDICAL IMAGING}

Developments in medical discipline have drastically changed health care over the last few years, allowing doctors to identify and treat diseases more effectively. But doctors, like any human beings, are also susceptible to inaccuracies. The credentialism of a doctor not only lie in the person's level of intelligence, but the manner they handle the problems of patients and the correlated type of health system that supports them (Lundervold \& Lundervold, 2019). In the physical world, Medical imaging has abundant resources of health data, which includes Magnetic Resonance Imaging (MRI), Xray, Positron Emission Tomography (PET), Computerized Tomography scan (CT scan), have provided doctors vast volumes of information(Liu et al., 2015).

Medical imaging is that the wide-ranging-spectrum name given to the cluster of procedures and methods designed for generating functional images, which are used for both clinical and scientific purposes. Medical image analysis is one among the leading substantial research within the field of medical, because of the results obtained by the evaluation which leads Medico professionals for analysis, therapy planning, and verification of administered treatment. Furthermore, modern developments in medical imaging techniques provided a major drop within the requirement for intrusive involvement in treatment of a spread of diseases or abnormalities (Aruna, 2020). Deep learning as an artificial intelligence method has demonstrated favorable outcomes in aiding disease analysis using CT images . Advancing after the solid feature learning ability, Deep Learning is capable of extract features that are correlated to medical outcomes from CT images automatically. Convolutional Neural Network (CNN) of deep learning is one of the most powerful way to for image representation and other structured data. These models perform classify the images based the self-learned image features using feature extraction Let us have a brief look at the basic building blocks of convolutional neural network in medical imaging.

\section{BUILDING BLOCKS OF CNN}

A Convolutional Neural Network is a certain form of artificial neural network designed at preserving spatial connections in the data, with extremely limited connections between the layers. The input to a $\mathrm{CNN}$ is assembled in a grid structure and then fed through layers that sustain these relationships, every single layer operates on a small region of the previous layer has been described in the Figure 2. CNN's can develop an extremely efficient interpretation of the input data. A CNN model has several layers of convolutions and activations, often combined with pooling layers, and is trained using backpropagation and gradient descent in the same way as for standard artificial neural networks. In addition, CNN's typically have fully connected layers at the end, which compute the final productions.

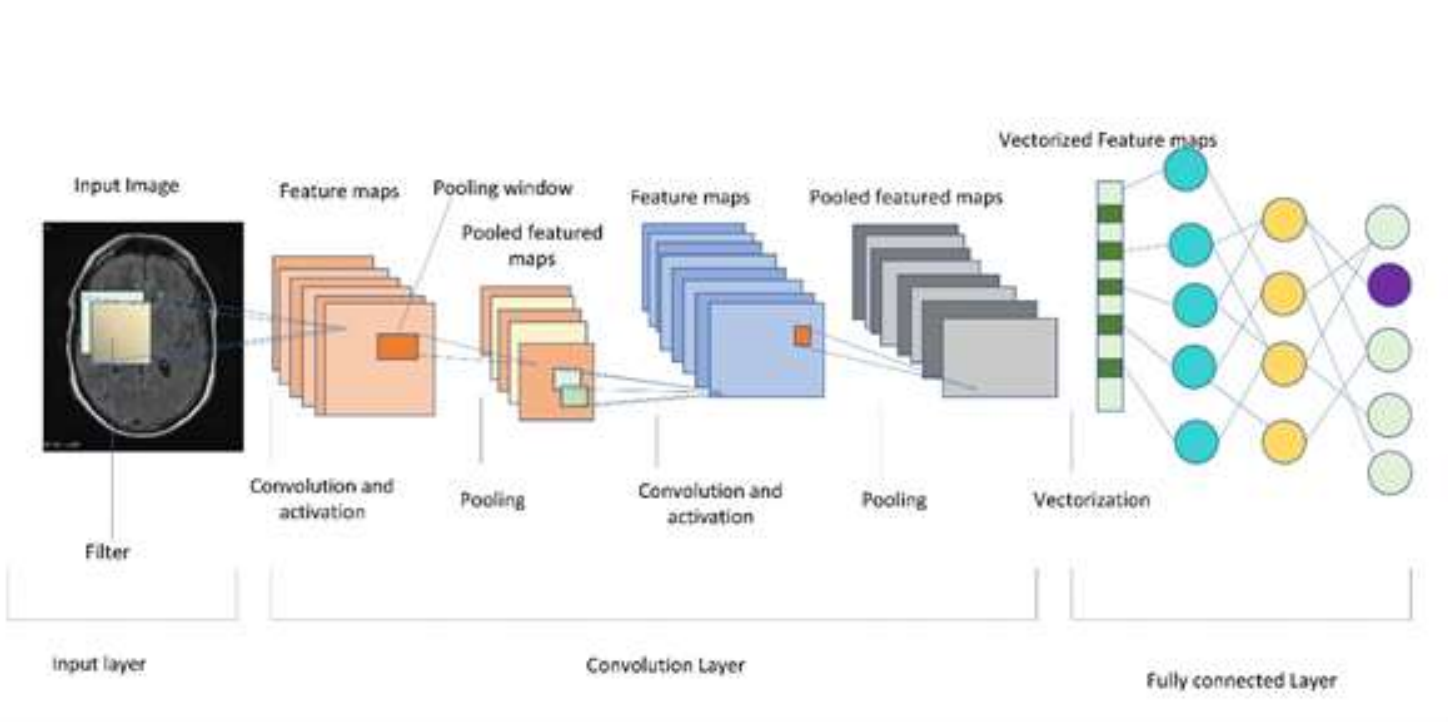

Figure 2. A Basic fully connected CNN architecture.

Convolutional layers: This layer is the initial layer that is employed to extract the various features from the input images. In this layer, the arithmetical operation of convolution is performed between the input image and a filter of a particular size $\mathrm{N} x \mathrm{~N}$. By sliding the filter on the input image, the dot product is taken among the filter and the parts of the input image with respect to the size of the filter $(\mathrm{N} x \mathrm{~N})$. The output is called as the Feature map which provides us knowledge 


\title{
International Journal of Advanced Research in Computer and Communication Engineering
}

\author{
Vol. 10, Issue 9, September 2021
}

DOI: $10.17148 /$ IJARCCE.2021.10904

about the image such as the corners and edges. This feature map is fed to the next layers to discover several other features of the input image.

Pooling layer: Every Single feature map produced by feeding the data all through one or more convolutional layer is then typically assembled in a pooling layer. Pooling operations take small grid regions as input and generate single figure for each area. The figure is usually calculated by employing the max function (max-pooling) or the average function (average pooling). Since a minor change of the input image effects in minor changes in the activation maps, the pooling layers gives the Convolutional neural network some translational invariance. A distinct way of bringing down the sampling effect of pooling is to use convolutions with enhanced stride lengths. Eliminating the pooling layers simplifies the network architecture without necessarily sacrificing performance.

Fully Connected Layer: The Fully Connected layer includes the weights and biases along of the neurons and is used to connect the neurons between two distinct layers. These layers are normally placed before the output layer and generate the last few layers of a CNN Architecture. In this, the input image from the preceding layers is flattened and fed to the Fully Connected layer. The flattened vector then withstands few more Fully Connected layers where the mathematical functions procedures typically take place. In this stage, the classification method begins to take place.

Dropout regularization: It is a straightforward notion that offered a massive boost in the performance of Convolution neural networks. By averaging quite a few models in an ensemble one tends to get better results than when using single models. Dropout is an averaging method based on stochastic sampling of neural networks. By accidentally removing neurons while training one ends up using marginally altered networks for every single batch of training data, and the weights of the trained network are fine-tuned based on optimization of multiple variations of the network.

Batch normalization: Batch normalization layers are more often placed after the activation layers, generating normalized activation maps by deducting the mean and dividing by the standard deviation for every training batch. Containing batch normalization layers forces the network to regularly change its activations to zero mean and unit standard deviation while the training batch hits these layers, which works as a regularize for the network, hustles up the training, and makes it less reliant on the cautious parameter initialization.

\section{DEEP LEARNING IN COVID-19 DIAGNOSIS}

Deep learning is a subset of Artificial intelligence which learns from the historical data to provide predictions for the future outcomes. Especially deep learning is an are based on self-learning and improving on its own by analyzing machine algorithms. Deep learning Models are trained by engaging a huge set of labeled data and neural network architectures that include numerous layers (Aruna, 2020). The laboratory analysis of biomedical and healthcare challenges helps health experts and scientists to discover out the new scope for helping the healthcare communities. The exposure of COVID-19 at an early stage and isolation of the involved people from others is the extremely crucial step in monitoring this pandemic due to high transmission of the virus. The Primary COVID-19 key indicator test for diagnosing the virus is rRT-PCR. But this test is time consuming and sometimes produces high rate of false negative results.

The recent swift spread of COVID-19 leads to exploring different methods for spotting coronavirus infection using medical imaging. Deep learning techniques in medical imaging have been used to process and analyze X-rays and computed tomography (CT), and PET to support the medical professional to forecast COVID-19 infection (Rawat et al., n.d.). Several research were introduced, focusing on the diagnosing coronavirus using different architectures of convolutional neural networks in a deep learning model were used to distinguish covid from non-covid-19 groups. For the analysis purpose, following models are employed which are formerly trained on ImageNet dataset which is a large dataset of about 14 million images of about 1000 different classes: AlexNet, VGGNET (VGG-1 and VGG-19), SqueezeNet, GoogleNet, MobileNet-V2, ResNet-18, ResNet-50, ResNet-101, and Xception has been clearly demonstrated in Figure 3. 
Vol. 10, Issue 9, September 2021

DOI: $10.17148 /$ IJARCCE.2021.10904

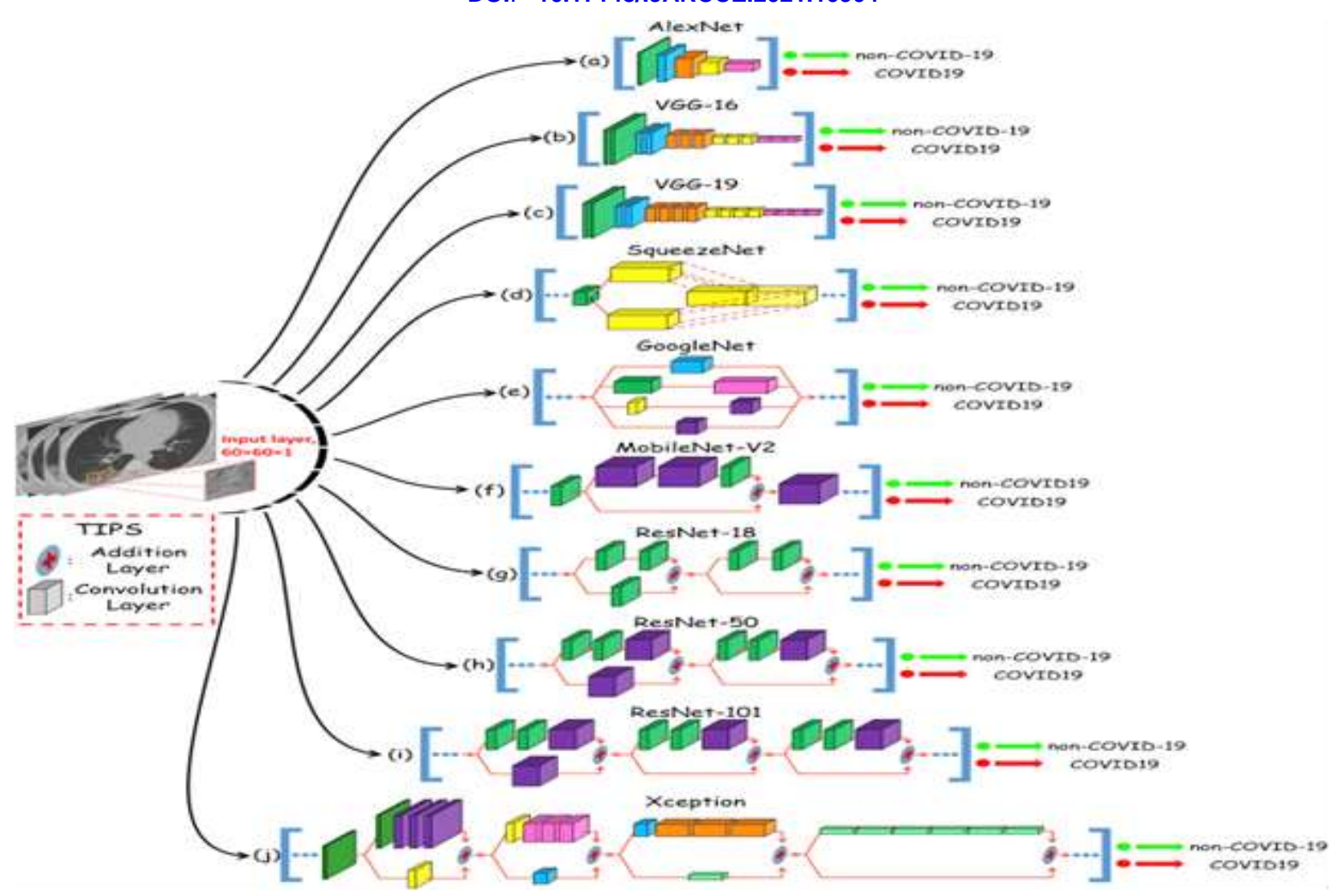

Figure 3. An overview of pre-trained neural networks architecture for Covid-19 diagnosis

Alex Net: Alex Net is a type of feedforward convolution neural network with 8-layer deep. It contains 5 convolution layers, 3 fully- connected layers. It was suggested by Alex Krizhevsky and trained on 1 million images to classify images into 1000 different classes. There are some additional features that makes, Alex Net more special in the performance of disease detection. ReLU Nonlinearity. Alex Net uses Rectified Linear Units (ReLU) instead of the tanh function which was able to reach $25 \%$ error on CIFAR-10 dataset six times faster than a CNN using tanh.

Multiple GPUs: Alex Net uses Multiple GPU's for reducing the training time. This can be achieved by splitting half of the model's neurons in one GPU and other half on another GPU.

Overlapping Pooling: Convolutional neural network conventionally pool outputs of nearby clusters of neurons with no overlapping. However, when the authors-initiated overlap, they noticed a reduction in error by about $0.5 \%$ and found that models with overlapping pooling generally find it harder to overfit.

The Overfitting Problem:Alex Net had sixty million parameters, a most important issue in terms of overfitting. Two methods Data Augmentation and Drop out were used to reduce overfitting.

VGGNet- VGG-16 AND VGG-19: VGG16 and VGG19 are usually called VGGNet that introduced the ImageNet Large Scale Visual Recognition Challenge (ILSVRC) assesses algorithms for object detection and image classification at a grand scale. It is named as VGG16 or VGG19 based on the number of layers deployed. They utilize only $3 \times 3$ convolution layer and max pooling. These models simply load up the layers on the right top. Evaluate of these models are compared based on the layer count using these two models. VGG19 has more three layers than VGG16 has been shown in Figure 4.

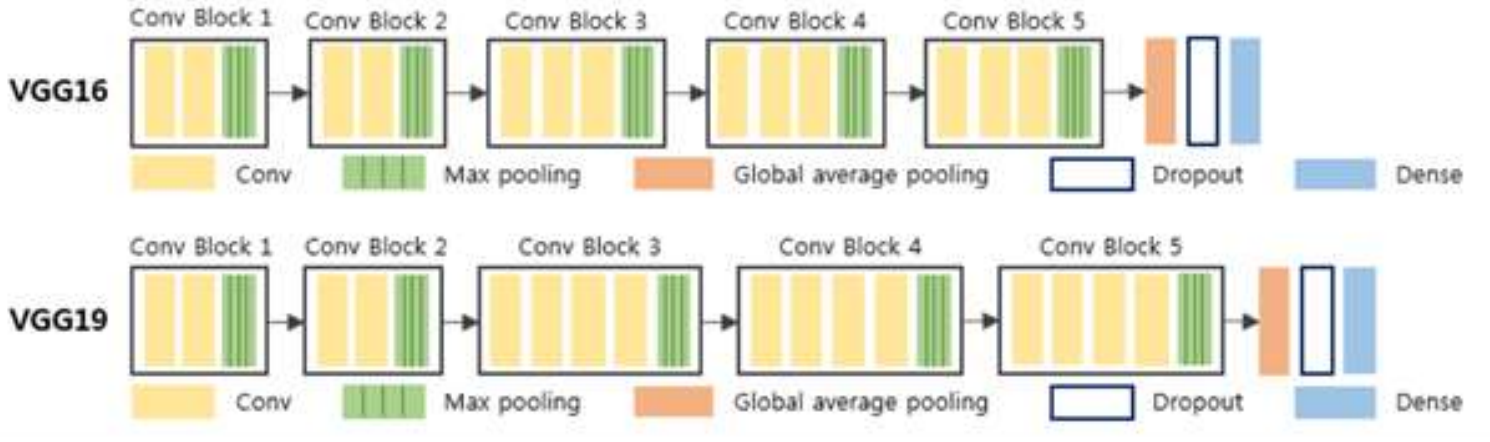

Figure 4. VGGNet basic architecture 
Vol. 10, Issue 9, September 2021

DOI: $10.17148 /$ IJARCCE.2021.10904

Inception V3: Inception v3 is a wider model than VGGNet. It is also introduced the ILSVRC and gained. This model is organized of inception component that incorporates convolution layers and pooling layer in parallel. Inception V3 module makes the model more trainable. The inception module is considered as a block. Even Though of a wider and deeper model, it has fewer parameter than VGGNet. Thus, this model converges extremely quickly demostarted in Figure 5.

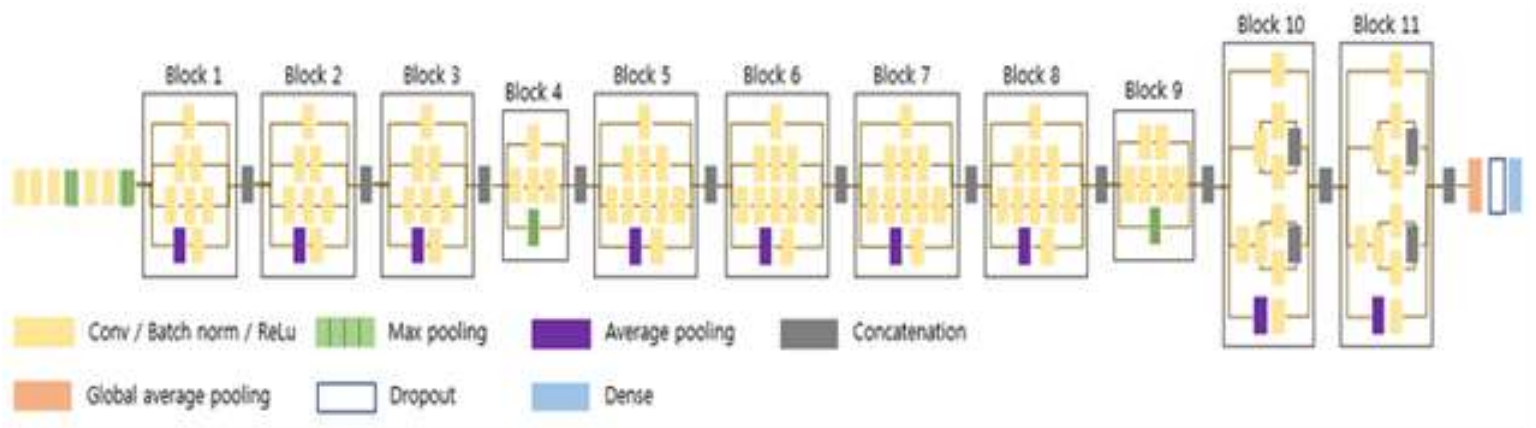

Figure 5. Inception V3 architecture

SqueezeNet: Squeeze Net is a single compact CNN with 18 deep learnable layers to classify images into 1000 different classes. It begins with a standalone convolution layer (conv1) has been shoen in Figure 6, followed by 8 Fire modules , ending with a final convolutional layer (conv10). Progressively boost the number of filters per fire module from the starting to the end of the network (Iandola et al., 2016).

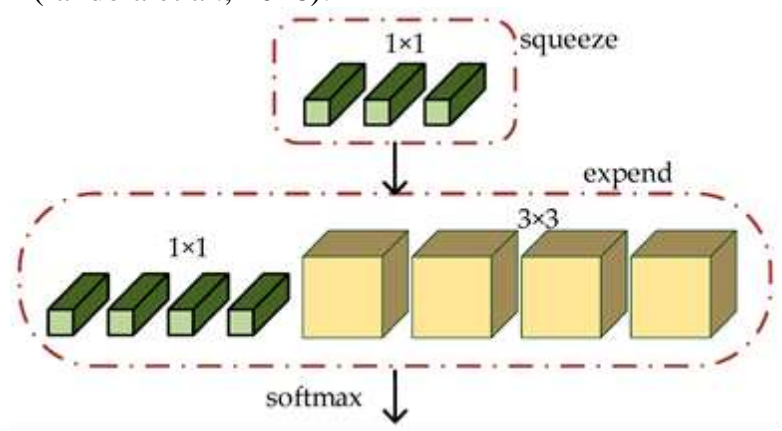

Figure 6.Layers of SqueezeNet architecture

GoogLeNet: This is a deep model which is used to solve most of the problems that larger networks faced, mainly through the Inception module's deployment. The network is 22-layers deep, starts with 3 convolution layers, pursued by 9 inception blocks, and ends with a fully attached layer. The inception block act as a core part of the GoogLeNet. Figure 7 shows the the architecture, which has been designed to be a driving force for the increased computational efficiency compared to a few of its predecessors or similar networks created at the time.

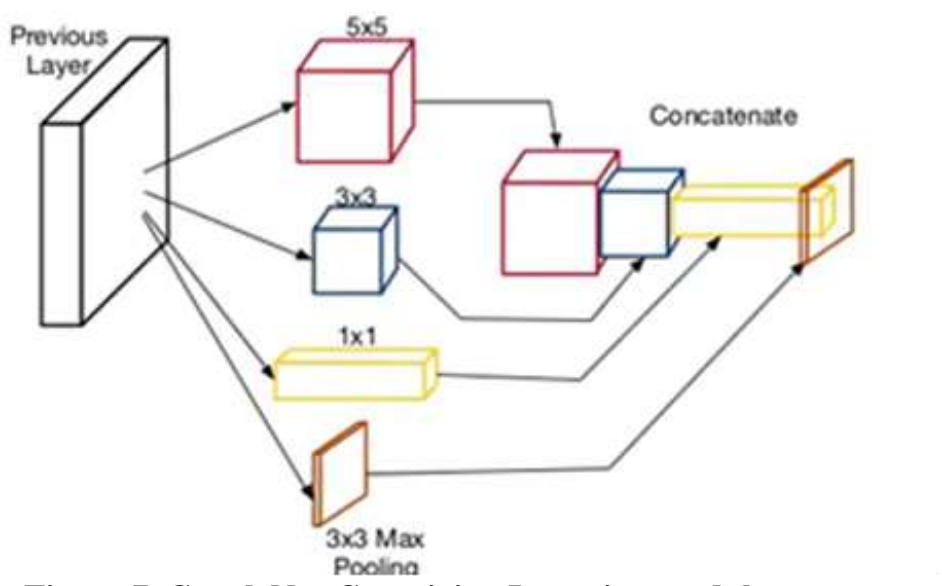

Figure 7. GoogleNet-Conceiving Inception module 
Vol. 10, Issue 9, September 2021

DOI: $10.17148 / I J A R C C E .2021 .10904$

MobileNet: It is a less load CNN with 53 layers deep of which 52 convolution and 1 fully connected layer. The major part design of the network is constructed on inverted residual and linear bottlenecks. The model starts with three convolution layers, followed by 16 inverted residual and linear bottleneck blocks and ends with 1 convolution layer and 1 fully connected layer. The idea of the inverted residual block is to remains as the main part of the network has been demonstrated in Figure 8.
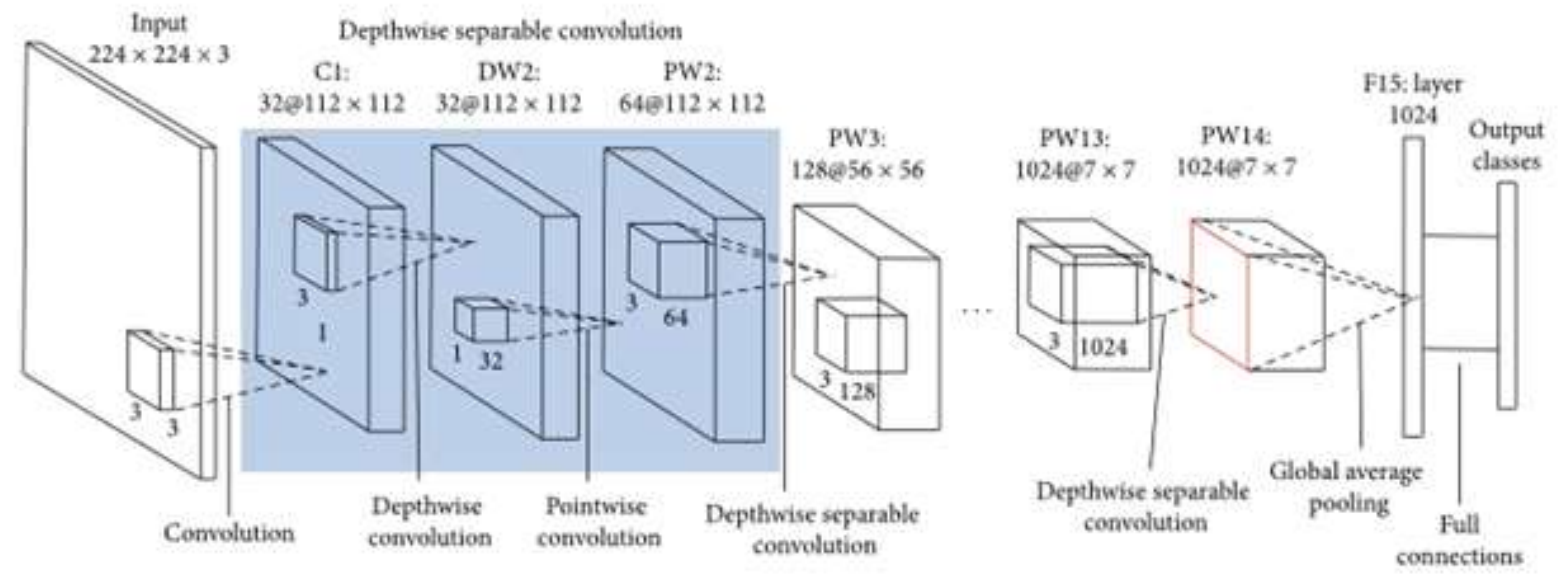

Figure 8. Basic architecture of MobileNet Convolutional Neural Network

ResNet: It is a type of deep neural network based on residual learning. This type of learning can simplify the training phase of networks by considering the layer inputs as a reference Figure 9. All forms of ResNet, ResNet-18, ResNet-50, and ResNet-101, are versions of ResNet which have their own particular residual block has been described in the Table 1.

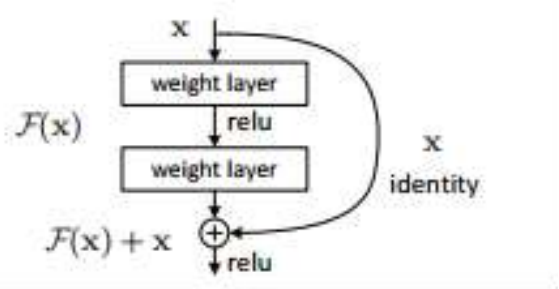

Figure 9. Residual block of ResNet architecture.

\begin{tabular}{|l|l|l|l|}
\hline SNO & Resnet Version & Deep & Residual \\
\hline $\mathbf{1}$ & ResNet-18 & 22 layers & 8 \\
\hline $\mathbf{2}$ & ResNet-50 & 50 & 16 \\
\hline $\mathbf{3}$ & ResNet-101. & 101 & 33 \\
\hline
\end{tabular}

Tabel 1. Layers of ResNet Convolutional neural networks.

Xception: This Model has been proposed by Francois Chollet, who is software engineer at Google Inc. In Xception architecture standard Inception modules are changed with depth wise Separable Convolutions in Figure 10. It begins with two convolution layers, followed by depth wise separable convolution layers, four convolution layers, and a fully connected layer. 


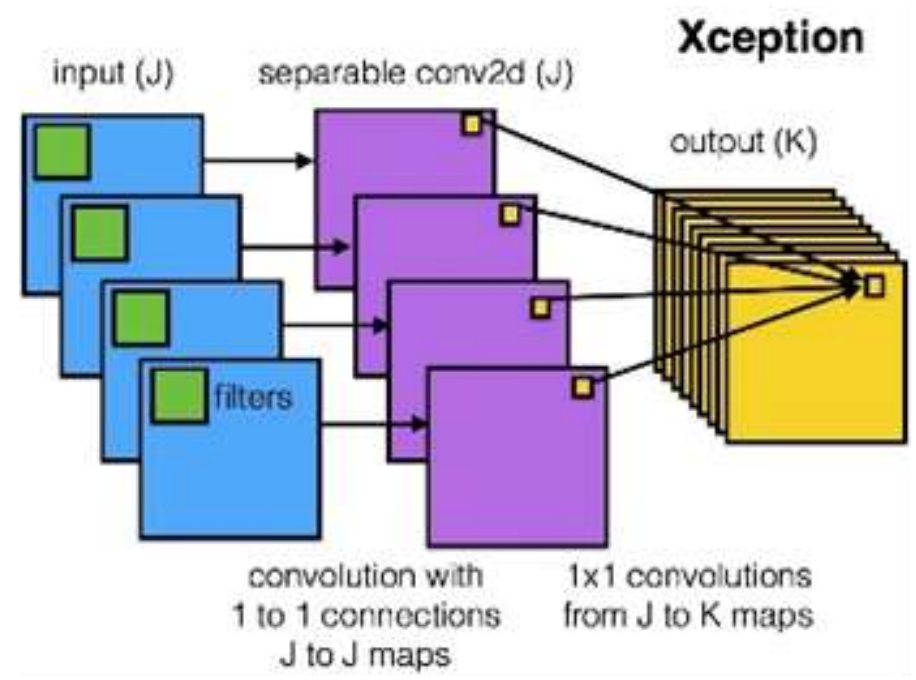

Figure 10. Xception: Deep Learning with Depthwise Separable Convolutions

\section{DEEP LEARNING IN VIRUS SEVERITY AND INFECTIVITY}

Covid-19 viral host prediction is an important task to ensure biosafety for changing viruses quickly. It is a challenging work to detect infectious viruses which are greater threat to mankind using bioinformatics systems. An approach was proposed to predict whether a virus can infect the human being directly utilizing next generation in order. Figure 11 shows the system, proved that Convolution Neural Network and Long short-term memory (LSTM) based architecture surpassed the more machine learning algorithms and simplified to taxonomical units with a partial error rate from those that are in the training phase (Bartoszewicz et al., 2021).

The nucleotide data is visualized in the convolutional filters. Finally, data are plotted to provide the insight for the behavior of the system to investigate the COVID-19 virus. A deep learning algorithm-based virus host prediction technique have been introduced to identify possible types of a viral host can infect a human with DNA input sequence (Guo et al., 2020). The obtained result showed that various vertebral infectious covid-19 has great force for infecting mankind. This system is also efficient for virus analysis and prevention in an early stage.

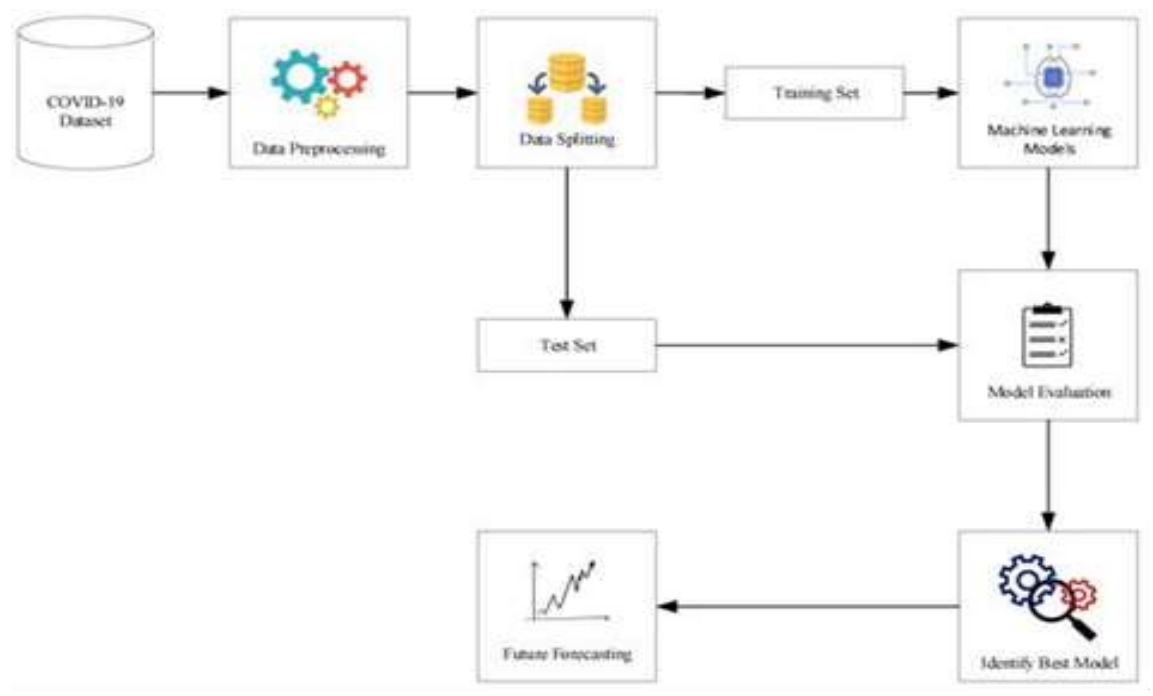

Figure 11. A deep-learning-based approach for severity analysis and monitoring the disease

\section{DEEP LEARNING FOR COVID-19 DISEASE TRACKING}

Deep learning procedures are often employed to trace the propagation of COVID-19 infection. Instead of using traditional medical imaging, depth camera footage can be used to analyze respirational patterns to forecast tachypnea (Wang et al., 2020). The scientists employed bidirectional Gated recurrent unit (GRU) method to prediction tachypnea that could be a 
Vol. 10, Issue 9, September 2021

DOI: $10.17148 / I J A R C C E .2021 .10904$

major investigative feature to provide large-scale diagnosis of COVID-19 patients. Smartphone sensors are employed to locate COVID-19 symptoms based on a deep learning algorithm to trace the COVID-19 pandemic. A $\alpha$-satellite AI driven system to detect geographical risk estimation at population levels. The substantial real-time data are used in deep neural networks models to discover the community opinions and calculate the risk level. A prediction model based on LSTM networks is developed to predict the movement of the COVID-19 outbreak in Canada (Chimmula \& Zhang, 2020). A combined LSTM and GRU model has been presented to assess positive, negative, death, and release cases of COVID-19 for approximating the situation of the present epidemic (Dutta \& Bandyopadhyay, 2020). Figure 12 demonstrates evidence, that can be applied to take accurate actions to monitor and control the covid-19 infection.

(a) Data Collection

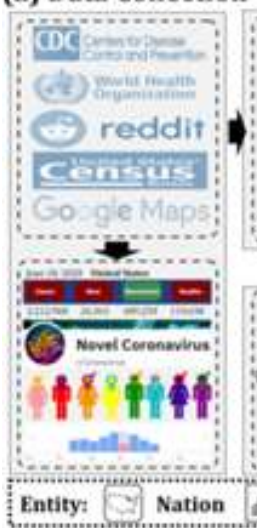

(b) AHIN Construction

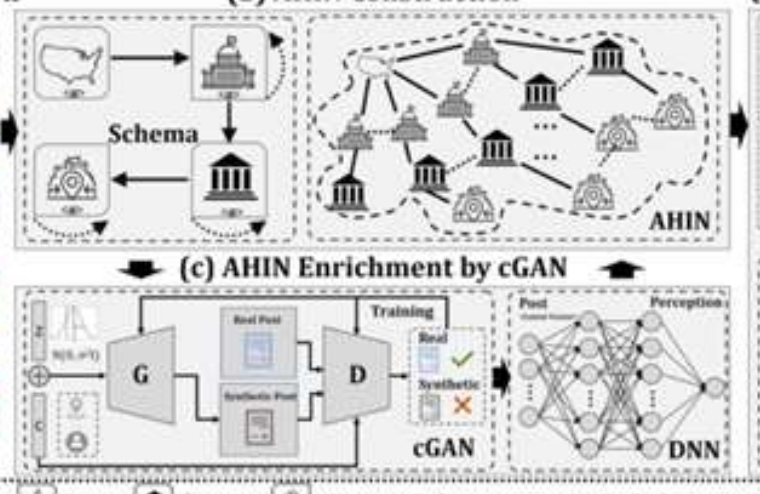

(d) Dynamic CoviD-19 Risk Assessment

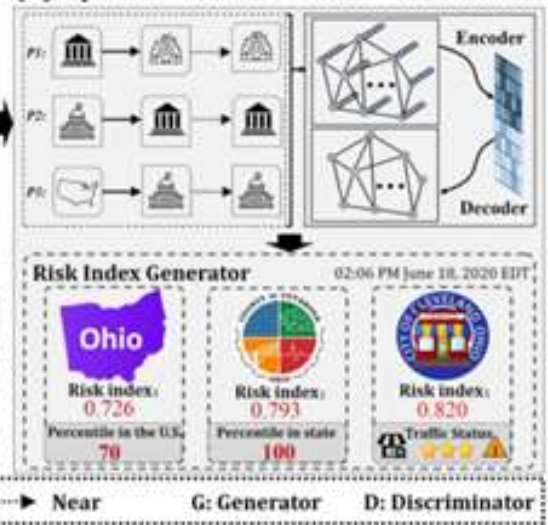

Figure 12.Covid-19 Disease tracking using Deep learning

\section{DEEP LEARNING FOR PROTEIN STRUCTURE PREDICTION}

Protein structure prediction can be used to define the 3D shape of a protein from its amino acid sequence. As the structure of a protein largely determines its function based on its sequence; however, protein structures can be tricky to decide by experimentation. Recently, employing genetic information has shown a dramatic progress.The protein structures can be predicted by analyzing the covariation in homologous sequences which are in contact of amino residues.

A neural network can be trained to make exact predictions of the distances between pairs of residues that provides more information of the structure than contact predictions. This information can be used to construct a potential of mean force that can precisely explain the shape of a protein. The resultant potential can be enhanced by a first-order iterative gradient descent algorithm to produce protein structures without difficult sampling procedures. The resultant system, named AlphaFold, accomplishes high accuracy, even for structures with a small number of homogenous sequences. Critical Assessment of Protein Structure Prediction created high accuracy structures for (24 out of 43) free modelling domains have been demonstrated in Figure 13.

The next best method used sampling and contact information, achieved the accuracy of (14 out of 43) domains. AlphaFold signifies a substantial improvement in protein-structure prediction. This increased accuracy is used to enable understandings into the function and malfunction of homologues proteins experimentally (Senior et al., 2020). This 
Vol. 10, Issue 9, September 2021

DOI: $10.17148 / I J A R C C E .2021 .10904$

would be beneficial to observe the functionality of coronavirus and also for the COVID-19 medicine development.

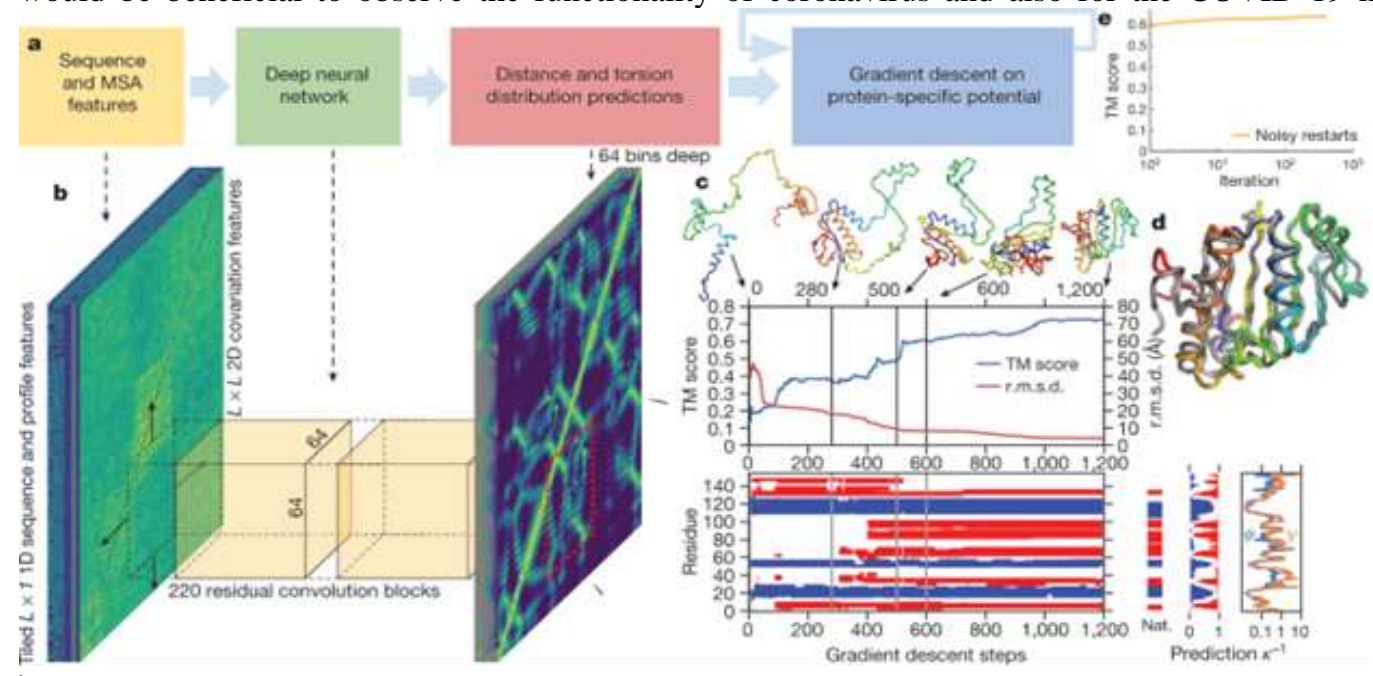

Figure 13. Protein Prediction using Deep Learning

\section{DEEPLEARNING FOR DRUG DISCOVERY/REPURPOSING}

A Phenotype-based combination screening has benefits over objective-based drug discovery which is impenetrable and lacks understanding of structure of drug action.A chemically Induced Dimerization (CID) gene expression shape provides a mechanistic signature of phenotypic response. The use of such data is constrained by their meagerness, inaccuracy and comparatively low output. Few techniques can present phenotype-based de novo chemical compound screening. A mechanism-driven neural network-based method, Docker Enumeration, Escalation of Privileges and Container Escapes (DeepCE) have been proposed (Pham et al., 2021), which uses a graph neural network and multiheaded attention mechanism to model gene chemical substructure and gene by gene connotation for predicting the variance gene structure profile distress by de novo chemicals. A ground-breaking data augmentation approach has been proposed which extracts useful data from inaccurate experiments in the L1000 dataset. The investigational findings show that DeepCE attains exceptional performances to state-of-the-art methods. The efficacy of gene representation summaries generated from DeepCE has been compared with monitored data for downstream categorization tasks. To verify the value of DeepCE, it has been applied to drug repurposing of COVID-19 and deliver unique lead combinations reliable with clinical evidence. DeepCE thus offers a possible strong framework for vigorous predictive modelling by applying noisy omics data and testing different chemicals for the variation of the systemic response to disease have been demonstrated in Figure 14.

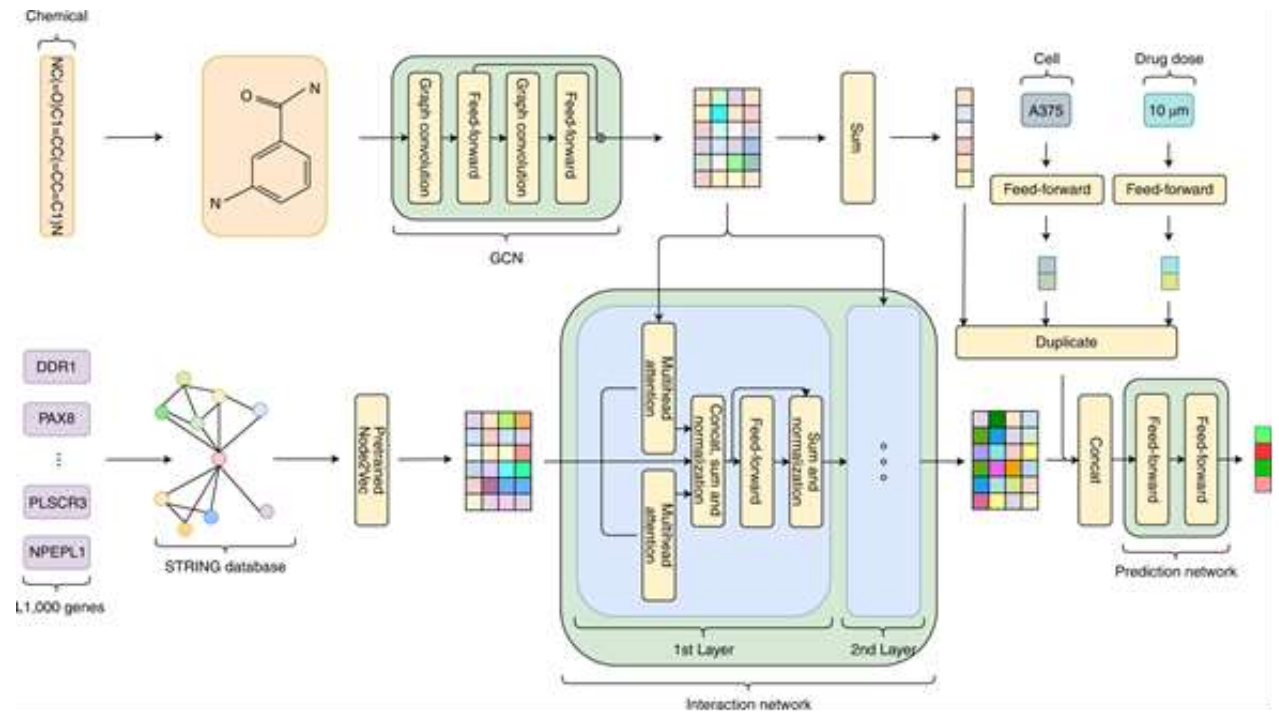

Figure 14. A data-driven drug repurposing framework of deep learning targeting COVID-19 


\section{International Journal of Advanced Research in Computer and Communication Engineering}

Vol. 10, Issue 9, September 2021

DOI: $10.17148 /$ IJARCCE.2021.10904

IX CONCLUSION

Deep Learning has been considered as excellent technique to provide intelligent solutions for combatting COVID-19. Influenced by numerous applications of Deep Learning for medical image processing in the past period, we have summarized recent efforts about the COVID- 19 epidemic for smart and healthy cities. Deep Learning has been used to reach several solutions for COVID-19 disruption, including virus outbreak prediction, a virus spread tracking, diagnosis and treatment, drug discovery and repurposing research. Despite favourable results, the successful use of Deep Learning to handle COVID-19 medical images still needs significant amount of time and effort along with close process between various parties from government, industry, and academic circles. In the End, we have considered a number of potential directions of Deep Learning applications for COVID-19 medical image processing. We think that the COVID-19 outbreak will be ending soon with help from Deep Learning and medical image processing techniques as well as several other technologies like biomedicine, data science, and mobile telecommunications. We also expect that our work is an excellent source of reference and be able to drive many new studies on Deep Learning and medical image processing in the struggle against the COVID-19.

\section{REFERENCES}

[1].About Variants of the Virus that Causes COVID-19 | CDC. (n.d.). Retrieved July 11, 2021, from https://www.cdc.gov/coronavirus/2019ncov/variants/variant.html

[2]. Ardakani, A. A., Kanafi, A. R., Acharya, U. R., Khadem, N., \& Mohammadi, A. (2020). Application of deep learning technique to manage COVID19 in routine clinical practice using CT images: Results of 10 convolutional neural networks. Computers in Biology and Medicine, 121 , 103795. https://doi.org/10.1016/J.COMPBIOMED.2020.103795

[3]. Bartoszewicz, J. M., Seidel, A., \& Renard, B. Y. (2021). Interpretable detection of novel human viruses from genome sequencing data. BioRxiv, 18, 2020.01.29.925354. https://doi.org/10.1101/2020.01.29.925354

[4]. Chimmula, V. K. R., \& Zhang, L. (2020). Time series forecasting of COVID-19 transmission in Canada using LSTM networks. Chaos, Solitons \& Fractals, 135, 109864. https://doi.org/10.1016/J.CHAOS.2020.109864

[5]. Dutta, S., \& Bandyopadhyay, S. K. (2020). Machine Learning Approach for Confirmation of COVID-19 Cases: Positive, Negative, Death and Release. MedRxiv, 2020.03.25.20043505. https://doi.org/10.1101/2020.03.25.20043505

[6]. G, Aruna. (2020). Application of Deep Learning in Medical Image Processing - A Comprehensive Review. Ijarcce, 9(6), 135-141. https://doi.org/10.17148/ijarcce.2020.9622

[7]. Guo, Q., Li, M., Wang, C., Wang, P., Fang, Z., tan, J., Wu, S., Xiao, Y., \& Zhu, H. (2020). Host and infectivity prediction of Wuhan 2019 novel coronavirus using deep learning algorithm. BioRxiv, 2020.01.21.914044. https://doi.org/10.1101/2020.01.21.914044

[8]. Iandola, F. N., Han, S., Moskewicz, M. W., Ashraf, K., Dally, W. J., \& Keutzer, K. (2016). SqueezeNet: AlexNet-level accuracy with 50x fewer parameters and $<0.5 M B$ model size. February, 0-13. http://arxiv.org/abs/1602.07360

[9]. Kumar, A., Gupta, P. K., \& Srivastava, A. (2020). A review of modern technologies for tackling COVID-19 pandemic. Diabetes \& Metabolic Syndrome: Clinical Research \& Reviews, 14(4), 569-573. https://doi.org/10.1016/J.DSX.2020.05.008

[10]. Liu, Y., Liu, S., \& Wang, Z. (2015). A general framework for image fusion based on multi-scale transform and sparse representation. Information Fusion, 24, 147-164. https://doi.org/10.1016/J.INFFUS.2014.09.004

[11]. Lundervold, A. S., \& Lundervold, A. (2019). An overview of deep learning in medical imaging focusing on MRI. In Zeitschrift fur Medizinische Physik (Vol. 29, Issue 2, pp. 102-127). Elsevier GmbH. https://doi.org/10.1016/j.zemedi.2018.11.002

[12]. Pham, T.-H., Qiu, Y., Zeng, J., Xie, L., \& Zhang, P. (2021). A deep learning framework for high-throughput mechanism-driven phenotype compound screening and its application to COVID-19 drug repurposing. Nature Machine Intelligence 2021 3:3, 3(3), $247-257$. https://doi.org/10.1038/s42256-020-00285-9

[13]. Rawat, R. M., Garg, S., Jain, N., \& Gupta, G. (n.d.). COVID-19 Detection using Convolutional Neural Network Architectures based upon Chest X-rays Images. https://doi.org/10.1109/ICICCS51141.2021.9432134

[14]. Rodriguez-Palacios, A. (2020). Modeling the Onset of Symptoms of COVID-19. Frontiers in Public Health|www.frontiersin.org, 473.

[15]. Senior, A. W., Evans, R., Jumper, J., Kirkpatrick, J., Sifre, L., Green, T., Qin, C., Žídek, A., Nelson, A. W. R., Bridgland, A., Penedones, H., Petersen, S., Simonyan, K., Crossan, S., Kohli, P., Jones, D. T., Silver, D., Kavukcuoglu, K., \& Hassabis, D. (2020). Improved protein structure prediction using potentials from deep learning. Nature 2020 577:7792, 577(7792), 706-710. https://doi.org/10.1038/s41586-019-1923-7

[16].Tracking SARS-CoV-2 variants. (n.d.). Retrieved July 11, 2021, from https://www.who.int/en/activities/tracking-SARS-CoV-2-variants/

[17]. Villalta, D., Martelli, P., Moratto, A., Salgarolo, V., Ligato, E., Conte, M., Giacomello, R., Pellis, T., De Rosa, R., Venturini, S., \& Crapis, M. (2021). Diagnostic performance of an automated chemiluminescence immunoassay for SARS-CoV-2 IgG and IgM antibodies detection: A real life experience. Practical Laboratory Medicine, 25, e00227. https://doi.org/10.1016/J.PLABM.2021.E00227

[18]. Wang, Y., Hu, M., Li, Q., Zhang, X.-P., Zhai, G., \& Yao, N. (2020). Abnormal respiratory patterns classifier may contribute to large-scale screening of people infected with COVID-19 in an accurate and unobtrusive manner. https://arxiv.org/abs/2002.05534v2

WHO lists additional COVID-19 vaccine for emergency use and issues interim policy recommendations. (n.d.). Retrieved July 11, 2021, from https://www.who.int/news/item/07-05-2021-who-lists-additional-covid-19-vaccine-for-emergency-use-and-issues-interim-policy-recommendations 